\title{
Unification of several distance functions and a common fixed point result
}

\author{
Mujahid Abbas ${ }^{1}$, Bashir Ali ${ }^{2}$ and Yusuf I Suleiman ${ }^{3 *}$
}

"Correspondence: yubram@yahoo.com

${ }^{3}$ Department of Mathematics, Kano University of Science and Technology, P.M.B. 3042, Wudil, Kano, Nigeria

Full list of author information is available at the end of the article

\begin{abstract}
In this work, we initiate the notions of dislocated- $A_{b}$-quasi-metric and $A_{b}$-quasi-metric-like spaces. Then we establish the existence of a common fixed point of weakly compatible mappings satisfying a contractive condition on a closed neighborhood of dislocated $A_{b}$-quasi-metric spaces. Some examples are given to show that these spaces are more general than various known comparable metric spaces. Our result unify, complement, and generalize various known results in the literature.
\end{abstract}

MSC: $47 \mathrm{H} 10 ; 47 \mathrm{H} 09 ; 54 \mathrm{H} 25$

Keywords: dislocated-quasi-metric spaces; A-metric spaces; coincidence points; fixed points; weakly compatible mappings

\section{Introduction and preliminaries}

In software engineering, algorithms are designed by means of recursive denotational specifications. The running time and the memory space of computing such algorithms are two important factors that determine the efficiency of the software.

Scott [1] used a $T_{0}$ model for lambda calculus to construct a system of logic. He then employed fixed point techniques as a suitable mathematical tool for program verifications in denotational semantics of programming languages.

Scott results were extended by Matthews [2] by defining a partial ordering on $T_{2}$ models. He introduced the notion of partial metric spaces and studied their essential topological properties. Matthews successfully reinforced Scott's fixed point techniques with a metric. His approach turned out to be very productive and attracted the attention of several researchers, who studied fixed point results on partial metric spaces.

Arshad et al. [3] proved the existence of some fixed point results for mappings satisfying a contractive condition in a closed neighborhood of a certain point in an ordered dislocated metric space.

The aim of this paper is to introduce a notion of generalized partial metric spaces called dislocated $A_{b}$-quasi-metric spaces. We study basic topological properties of dislocated $A_{b}$ quasi-metric spaces and provide some examples to support the concepts defined herein. We also obtain common fixed point results of weakly compatible mappings satisfying local contractive condition in such spaces. Our results unify, improve, and generalize several comparable results in [4] and [3].

(c) 2016 Abbas et al. This article is distributed under the terms of the Creative Commons Attribution 4.0 International License (http://creativecommons.org/licenses/by/4.0/), which permits unrestricted use, distribution, and reproduction in any medium, provided you give appropriate credit to the original author(s) and the source, provide a link to the Creative Commons license, and indicate if changes were made. 
In the sequel, the letters $\mathbb{N}, \mathbb{N}_{0}, \mathbb{R}$, and $\mathbb{R}^{+}$denote the sets of positive integers, nonnegative integers, real numbers, and positive real numbers, respectively.

Definition 1.1 Let $X$ be a nonempty set, and $s \geq 1$ a given real number. A function $A_{b}$ : $X^{n} \rightarrow[0, \infty)$ is called a dislocated $A_{b}$-quasi-metric on $X$ if for any $a, x_{i} \in X, i=1,2, \ldots, n$, $n \geq 3$, the following conditions hold:

$$
\begin{aligned}
& \left(A_{b_{1}}\right) A_{b}\left(x_{1}, x_{2}, x_{3}, \ldots, x_{n-1}, x_{n}\right)=A_{b}\left(x_{n}, x_{n-1}, x_{n-2}, \ldots, x_{2}, x_{1}\right)=0 \text { implies that } x_{1}=x_{2}=x_{3}= \\
& \cdots=x_{n-1}=x_{n} \\
& \left(A_{b_{2}}\right) \\
& A_{b}\left(x_{1}, x_{2}, x_{3}, \ldots, x_{n-1}, x_{n}\right) \\
& \leq s\left[A_{b}\left(x_{1}, x_{1}, x_{1}, \ldots,\left(x_{1}\right)_{(n-1)}, a\right)\right. \\
& +A_{b}\left(x_{2}, x_{2}, x_{2}, \ldots,\left(x_{2}\right)_{(n-1)}, a\right) \\
& +A_{b}\left(x_{3}, x_{3}, x_{3}, \ldots,\left(x_{3}\right)_{(n-1)}, a\right) \\
& \vdots \\
& +A_{b}\left(x_{(n-1)}, x_{(n-1)}, x_{(n-1)}, \ldots,\left(x_{(n-1)}\right)_{(n-1)}, a\right) \\
& \left.+A_{b}\left(x_{n}, x_{n}, x_{n}, \ldots,\left(x_{n}\right)_{(n-1)}, a\right)\right] \text {. }
\end{aligned}
$$

The pair $\left(X, A_{b}\right)$ is called a dislocated $A_{b}$-quasi-metric space with coefficient $s$.

Lemma 1.2 Let $\left(X, A_{b}\right)$ be a dislocated $A_{b}$-quasi-metric space with coefficient $s \geq 1$. Then $A_{b}(x, x, x, \ldots, x, y)=A_{b}(y, y, y, \ldots, y, x)$ for all $x, y \in X$.

Example 1.3 Let $X=\mathbb{R}$. Define the function $A_{b}: X^{n} \rightarrow[0, \infty)$ by

$$
\begin{aligned}
A_{b}\left(x_{1}, x_{2}, x_{3}, \ldots, x_{n-1}, x_{n}\right)= & \left|2 x_{1}-x_{2}\right|^{2}+\left|2 x_{1}-x_{3}\right|^{2}+\cdots+\left|2 x_{1}-x_{n}\right|^{2} \\
& +\left|2 x_{2}-x_{3}\right|^{2}+\left|2 x_{2}-x_{4}\right|^{2}+\cdots+\left|2 x_{2}-x_{n}\right|^{2} \\
& \vdots \\
& +\left|2 x_{n-2}-x_{n-1}\right|^{2}+\left|2 x_{n-2}-x_{n}\right|^{2} \\
& +\left|2 x_{n-1}-x_{n}\right|^{2} .
\end{aligned}
$$

Indeed, if $A_{b}\left(x_{1}, x_{2}, x_{3}, \ldots, x_{n-1}, x_{n}\right)=A_{b}\left(x_{n}, x_{n-1}, x_{n-2}, \ldots, x_{2}, x_{1}\right)=0$, then $x_{1}=x_{2}=x_{3}=$ $\cdots=x_{n-1}=x_{n}$. Note that

$$
\begin{aligned}
A_{b}\left(x_{1}, x_{2}, x_{3}, \ldots, x_{n-1}, x_{n}\right)= & \left|2 x_{1}-x_{2}\right|^{2}+\left|2 x_{1}-x_{3}\right|^{2}+\cdots+\left|2 x_{1}-x_{n}\right|^{2} \\
& +\left|2 x_{2}-x_{3}\right|^{2}+\left|2 x_{2}-x_{4}\right|^{2}+\cdots+\left|2 x_{2}-x_{n}\right|^{2} \\
& \vdots \\
& +\left|2 x_{n-2}-x_{n-1}\right|^{2}+\left|2 x_{n-2}-x_{n}\right|^{2} \\
& +\left|2 x_{n-1}-x_{n}\right|^{2} \\
\leq & \left(\left|2 x_{1}-a\right|+\left|x_{2}-a\right|\right)^{2}
\end{aligned}
$$




$$
\begin{aligned}
& +\left(\left|2 x_{1}-a\right|+\left|x_{3}-a\right|\right)^{2} \\
& +\cdots+\left(\left|2 x_{1}-a\right|+\left|x_{n}-a\right|\right)^{2} \\
& +\left(\left|2 x_{2}-a\right|+\left|x_{3}-a\right|\right)^{2} \\
& +\left(\left|2 x_{2}-a\right|+\left|x_{4}-a\right|\right)^{2} \\
& +\cdots+\left(\left|2 x_{2}-a\right|+\left|x_{n}-a\right|\right)^{2} \\
& \vdots \\
& +\left(\left|2 x_{n-2}-a\right|+\left|x_{n-1}-a\right|\right)^{2} \\
& +\left(\left|2 x_{n-2}-a\right|+\left|x_{n}-a\right|\right)^{2} \\
& +\left(\left|2 x_{n-1}-a\right|+\left|x_{n}-a\right|\right)^{2} \\
& \leq 2\left[A_{b}\left(x_{1}, x_{1}, x_{1}, \ldots,\left(x_{1}\right)_{(n-1)}, a\right)\right. \\
& +A_{b}\left(x_{2}, x_{2}, x_{2}, \ldots,\left(x_{2}\right)_{(n-1)}, a\right) \\
& +A_{b}\left(x_{3}, x_{3}, x_{3}, \ldots,\left(x_{3}\right)_{(n-1)}, a\right) \\
& \vdots \\
& + \\
& +A_{b}\left(x_{(n-1)}, x_{(n-1)}, x_{(n-1)}, \ldots,\left(x_{(n-1)}\right)_{(n-1)}, a\right) \\
& \left.+A_{b}\left(x_{n}, x_{n}, x_{n}, \ldots,\left(x_{n}\right)_{(n-1)}, a\right)\right] .
\end{aligned}
$$

Hence, $\left(X, A_{b}\right)$ is a dislocated $A_{b}$-quasi-metric space with coefficient $s=2$.

Example 1.4 Let $X=\mathbb{R}$. Consider the dislocated $A_{b}$-quasi-metric on $X^{3}$ given by

$$
A_{b}\left(x_{1}, x_{2}, x_{3}\right)=\left|2 x_{1}-x_{2}\right|^{2}+\left|2 x_{1}-x_{3}\right|^{2}+\left|2 x_{2}-x_{3}\right|^{2} .
$$

If $x_{1}=4, x_{2}=6$, and $x_{3}=10$, then $A_{b}(4,6,10) \neq A_{b}(10,6,4)$ implies that $\left(X, A_{b}\right)$ is not a $G_{p_{b}}$-metric space [5].

Definition 1.5 Let $\left(X, A_{b}\right)$ be a dislocated $A_{b}$-quasi-metric space with $s \geq 1, x_{0} \in X$, and $r$ a positive real number $r$. The set

$$
\overline{B\left(x_{0}, r\right)}=\left\{y \in X: A_{b}\left(y, y, y, \ldots, y, x_{0}\right) \leq r\right\}
$$

is called a closed ball centered at $x_{0}$ with radius $r$.

Definition 1.6 A subset $G$ of a dislocated $A_{b}$-quasi-metric space $\left(X, A_{b}\right)$ is said to be an open set if for each $x \in G$, there exists a positive real number $r$ such that $B(x, r) \subseteq G$. A set $F \subseteq X$ is called closed if $X \backslash F$ is open. The collection of open sets in a dislocated $A_{b}$-quasimetric space $\left(X, A_{b}\right)$ is called the topology induced by the metric $A_{b}$.

Lemma 1.7 Let $\left(X, A_{b}\right)$ be a dislocated $A_{b}$-quasi-metric space with $s \geq 1$. Then for all $x, y \in X$, we have

$$
A_{b}(x, x, x, \ldots, x, z) \leq s\left[(n-1) A_{b}(x, x, x, \ldots, x, y)+A_{b}(y, y, y, \ldots, y, z)\right] .
$$


Definition 1.8 Let $\left(X, A_{b}\right)$ be a dislocated $A_{b}$-quasi-metric space with $s \geq 1$ and $n \geq 3$. A sequence $\left\{x_{k}\right\}$ in $X$ is said to be convergent in $X$ if there exists a point $x \in X$ such that $\lim _{k \rightarrow \infty} A_{b}\left(x_{k}, x_{k}, x_{k}, \ldots, x_{k}, x\right)=0$. In this case, we write $\lim _{k \rightarrow \infty} x_{k}=x$.

Lemma 1.9 Let $\left(X, A_{b}\right)$ be a dislocated $A_{b}$-quasi-metric space with $s \geq 1$ and $n \geq 3$. The limit of a convergent sequence $\left\{x_{k}\right\}$ in $X$ is unique.

Proof Suppose that $\left\{x_{k}\right\}$ converges to $x$ and $y$. Then given $\epsilon>0$, there exist $N_{1}, N_{2} \in \mathbb{N}$ such that

$$
A_{b}\left(x_{k}, x_{k}, x_{k}, \ldots, x_{k}, x\right)<\frac{\epsilon}{2(n-1) s}
$$

for every $k \geq N_{1}$ and

$$
A_{b}\left(x_{k}, x_{k}, x_{k}, \ldots, x_{k}, y\right)<\frac{\epsilon}{2 s}
$$

for every $k \geq N_{2}$. Choose $N=\max \left\{N_{1}, N_{2}\right\}$. Note that

$$
\begin{aligned}
& A_{b}(x, x, x, \ldots, x, y) \\
& \quad \leq s\left[(n-1) A_{b}\left(x, x, x, \ldots, x, x_{k}\right)+A_{b}\left(x_{k}, x_{k}, x_{k}, \ldots, x_{k}, y\right)\right] \\
& \quad=s\left[(n-1) A_{b}\left(x_{k}, x_{k}, x_{k}, \ldots, x_{k}, x\right)+A_{b}\left(x_{k}, x_{k}, x_{k}, \ldots, x_{k}, y\right)\right] \\
& \quad<s\left[(n-1) \times \frac{\epsilon}{2(n-1) s}+\frac{\epsilon}{2 s}\right]=\epsilon
\end{aligned}
$$

for every $k \geq N$. Since $\epsilon$ is arbitrary, $A_{b}(x, x, x, \ldots, x, y)=A_{b}(y, y, y, \ldots, y, x)=0$, and hence $x=y$.

Definition 1.10 Let $\left(X, A_{b}\right)$ be a dislocated $A_{b}$-quasi-metric space with $s \geq 1$ and $n \geq 3$. A sequence $\left\{x_{k}\right\}$ in $X$ is called Cauchy if $\lim _{k, m \rightarrow \infty} A_{b}\left(x_{k}, x_{k}, x_{k}, \ldots, x_{k}, x_{m}\right)=0$, that is, if for each $\epsilon \geq 0$, there exists $N \in \mathbb{N}$ such that for all $k, m \geq N$, we have $A_{b}\left(x_{k}, x_{k}, x_{k}, \ldots, x_{k}\right.$, $\left.x_{m}\right) \leq \epsilon$.

Lemma 1.11 Every convergent sequence in a dislocated $A_{b}$-quasi-metric space is a Cauchy sequence.

Remark 1.12 The converse of Lemma 1.11 does not hold in general. Let $X=\mathbb{Q}$ (the set of rational numbers), and let $A_{b}$ be a dislocated $A_{b}$-quasi-metric defined in Example 1.18. Let $\left\{x_{k}\right\}$ be a sequence defined by $x_{k}=\left(1+\frac{1}{k}\right)^{k}$. Note that

$$
\begin{aligned}
A_{b}\left(x_{k}, x_{k}, x_{k}, \ldots, x_{k}, x_{m}\right) & =s(n-1)\left|x_{k}-x_{m}\right|^{2} \\
& =s(n-1)\left|\left(1+\frac{1}{k}\right)^{k}-\left(1+\frac{1}{m}\right)^{m}\right|^{2} \rightarrow 0
\end{aligned}
$$

as $k, m \longrightarrow \infty$. Thus, $\left\{x_{k}\right\}$ is a Cauchy sequence. But $x_{k} \longrightarrow e$ as $k \longrightarrow \infty$. Hence, $\left\{x_{k}\right\}$ is not convergent in $\mathbb{Q}$. 
Definition 1.13 A dislocated $A_{b}$-quasi-metric space $X$ is said to be complete if every Cauchy sequence in $\left(X, A_{b}\right)$ is convergent.

Definition 1.14 Let $\left(X, A_{b_{X}}\right)$ and $\left(Y, A_{b_{Y}}\right)$ be dislocated $A_{b}$-quasi-metric spaces with $s \geq 1$ and $n \geq 3$. A function $f: X \rightarrow Y$ is called continuous if, given $x \in X$ and $\epsilon>0$, we can find a $\delta_{(x, \epsilon)}>0$ such that $A_{b_{Y}}(f(x), f(x), \ldots, f(x), f(y))<\epsilon$ whenever $A_{b_{X}}(x, x, \ldots, x, y)<\delta_{(x, \epsilon)}$.

Theorem 1.15 Let $\left(X, A_{b_{X}}\right)$ and $\left(Y, A_{b_{Y}}\right)$ be dislocated $A_{b}$-quasi-metric spaces with $s \geq$ 1 and $n \geq 3$. A function $f: X \rightarrow Y$ is continuous at a point $x_{0} \in X$ if and only if it is sequentially continuous at $x_{0}$.

Lemma 1.16 Let $\left(X, A_{b}\right)$ be a dislocated $A_{b}$-quasi-metric space. Then the function $A_{b}(x, x$, $x, \ldots, x, y)$ is continuous in all of its arguments. In other words, if there exist sequences $\left\{x_{k}\right\}$ and $\left\{y_{k}\right\}$ such that $\lim _{k \rightarrow \infty} x_{k}=x$ and $\lim _{k \rightarrow \infty} y_{k}=y$, then $\lim _{k \rightarrow \infty} A_{b}\left(x_{k}, x_{k}, x_{k}, \ldots, x_{k}, y_{k}\right)=$ $A_{b}(x, x, x, \ldots, x, y)$ and $\lim _{k \rightarrow \infty} A_{b}\left(y_{k}, y_{k}, y_{k}, \ldots, y_{k}, x_{k}\right)=A_{b}(y, y, y, \ldots, y, x)$.

Definition 1.17 Let $X$ be a nonempty set, and $s \geq 1$ a given real number. A function $A_{b}$ : $X^{n} \rightarrow[0, \infty)$ is called $A_{b}$-quasi-metric-like on $X$ if for any $a, x_{i} \in X, i=1,2, \ldots, n, n \geq 3$, the following conditions hold:

$$
\begin{aligned}
& \left(A_{b_{1}}\right) A_{b}\left(x_{1}, x_{2}, x_{3}, \ldots, x_{n-1}, x_{n}\right)=0 \text { implies } x_{1}=x_{2}=x_{3}=\cdots=x_{n-1}=x_{n} \text {; } \\
& \left(A_{b_{2}}\right) \\
& A_{b}\left(x_{1}, x_{2}, x_{3}, \ldots, x_{n-1}, x_{n}\right) \\
& \leq s\left[A_{b}\left(x_{1}, x_{1}, x_{1}, \ldots,\left(x_{1}\right)_{(n-1)}, a\right)\right. \\
& +A_{b}\left(x_{2}, x_{2}, x_{2}, \ldots,\left(x_{2}\right)_{(n-1)}, a\right) \\
& +A_{b}\left(x_{3}, x_{3}, x_{3}, \ldots,\left(x_{3}\right)_{(n-1)}, a\right) \\
& \vdots \\
& +A_{b}\left(x_{(n-1)}, x_{(n-1)}, x_{(n-1)}, \ldots,\left(x_{(n-1)}\right)_{(n-1)}, a\right) \\
& \left.+A_{b}\left(x_{n}, x_{n}, x_{n}, \ldots,\left(x_{n}\right)_{(n-1)}, a\right)\right] \text {. }
\end{aligned}
$$

The pair $\left(X, A_{b}\right)$ is called an $A_{b}$-quasi-metric-like space.

Example 1.18 Let $X=\mathbb{R}$. Define the function $A_{b}: X^{n} \rightarrow[0, \infty)$ by

$$
\begin{aligned}
A_{b}\left(x_{1}, x_{2}, x_{3}, \ldots, x_{n-1}, x_{n}\right)= & \left|x_{1}-x_{2}\right|^{2}+\left|x_{1}-x_{3}\right|^{2}+\cdots+\left|x_{1}-x_{n}\right|^{2} \\
& +\left|x_{2}-x_{3}\right|^{2}+\left|x_{2}-x_{4}\right|^{2}+\cdots+\left|x_{2}-x_{n}\right|^{2} \\
& \vdots \\
& +\left|x_{n-2}-x_{n-1}\right|^{2}+\left|x_{n-2}-x_{n}\right|^{2} \\
& +\left|x_{n-1}-x_{n}\right|^{2} .
\end{aligned}
$$

Then $\left(X, A_{b}\right)$ is an $A_{b}$-quasi-metric-like space with coefficient $s=2$. Indeed, if $n=3$, then we have

$$
A_{b}\left(x_{1}, x_{2}, x_{3}\right)=\left|x_{1}-x_{2}\right|^{2}+\left|x_{1}-x_{3}\right|^{2}+\left|x_{2}-x_{3}\right|^{2} .
$$


Note that $A_{b}\left(x_{1}, x_{2}, x_{3}\right)=0$ implies that $x_{1}=x_{2}=x_{3}$. Also,

$$
\begin{aligned}
A_{b}\left(x_{1}, x_{2}, x_{3}\right)= & \left|x_{1}-x_{2}\right|^{2}+\left|x_{1}-x_{3}\right|^{2}+\left|x_{2}-x_{3}\right|^{2} \\
= & \left|x_{1}-a+a-x_{2}\right|^{2}+\left|x_{1}-a+a-x_{3}\right|^{2} \\
& +\left|x_{2}-a+a-x_{3}\right|^{2} \\
\leq & \left(\left|x_{1}-a\right|+\left|x_{2}-a\right|\right)^{2}+\left(\left|x_{1}-a\right|+\left|x_{3}-a\right|\right)^{2} \\
& +\left(\left|x_{2}-a\right|+\left|x_{3}-a\right|\right)^{2} \\
= & \left|x_{1}-a\right|^{2}+2\left|x_{1}-a\right|\left|x_{2}-a\right|+\left|x_{2}-a\right|^{2} \\
& +\left|x_{1}-a\right|^{2}+2\left|x_{1}-a\right|\left|x_{3}-a\right|+\left|x_{3}-a\right|^{2} \\
& +\left|x_{2}-a\right|^{2}+2\left|x_{2}-a\right|\left|x_{3}-a\right|+\left|x_{3}-a\right|^{2} \\
\leq & 2\left[2\left|x_{1}-a\right|^{2}+2\left|x_{2}-a\right|^{2}+2\left|x_{3}-a\right|^{2}\right] \\
= & 2\left[A_{b}\left(x_{1}, x_{1}, a\right)+A_{b}\left(x_{2}, x_{2}, a\right)+A_{b}\left(x_{3}, x_{3}, a\right)\right] .
\end{aligned}
$$

Thus, $\left(X, A_{b}\right)$ is an $A_{b}$-quasi-metric-like space with coefficient $s=2$.

Proposition 1.19 If $\left(X, A_{b}\right)$ is a quasi-metric-like space, then it is a dislocated $A_{b}$-quasimetric space, but the converse does not hold in general.

Proof Let $\left(X, A_{b}\right)$ be an $A_{b}$-quasi-metric-like space with coefficient $s \geq 1$. Then $A_{b}\left(x_{1}, x_{2}\right.$, $\left.x_{3}, \ldots, x_{n-1}, x_{n}\right)=0$ implies that $x_{1}=x_{2}=x_{3}=\cdots=x_{n-1}=x_{n}$, which in turn gives that $A_{b}\left(x_{n}, x_{n-1}, x_{n-2}, \ldots, x_{2}, x_{1}\right)=0$.

Let $\left(X, A_{b}\right)$ be a dislocated $A_{b}$-quasi-metric space considered in Example 1.3. Since $A_{b}\left(x_{1}, x_{2}, x_{3}, \ldots, x_{n-1}, x_{n}\right)=0$ does not imply $x_{1}=x_{2}=x_{3}=\cdots=x_{n-1}=x_{n},\left(X, A_{b}\right)$ is not an $A_{b}$-quasi-metric-like space.

Example 1.20 Let $X=\mathbb{R}$. Define the function $A_{b}: X^{2} \rightarrow[0, \infty)$ by

$$
A_{b}\left(x_{1}, x_{2}\right)=\left|2 x_{1}-x_{2}\right|^{2} .
$$

Then $\left(X, A_{b}\right)$ is a dislocated quasi- $b$-metric space.

Definition 1.21 Let $(X, d)$ be a metric space, and $f, g: X \rightarrow X$. The pair $(f, g)$ is said to be weakly compatible on $X$ if $f$ and $g$ commute at their coincidence points, that is, if $f x=g x$ for some $x \in X$, then $g f x=f g x$.

\section{Main results}

In this section, we obtain a common fixed point result for mappings satisfying generalized local contractive condition in the setup of dislocated $A_{b}$-quasi-metric space $X$. We start with the following result.

Theorem 2.1 Letf, $g, T$, and $S$ be self mappings on a dislocated $A_{b}$-quasi-metric space $X$ with $s \geq 1, x_{0}, x_{1} \in X, y_{0}=S x_{0}=g x_{1}$, and $r>1$. Suppose that $S(Y) \subseteq g(Y), T(Y) \subseteq f(Y)$, and one of $S(Y), g(Y), T(Y)$, or $f(Y)$ is a complete subspace of $Y$, where $Y=\overline{B\left(y_{0}, r\right)}$. Suppose 
that there exists $\alpha \in\left[0, \frac{1}{2}\right)$ such that $0 \leq \gamma=(n-1) s \alpha<1$ with $0<\frac{\gamma}{1-\gamma}<1$ and for any $x, y \in Y$, we have

$$
A_{b}(S x, S x, \ldots, S x, T y) \leq \alpha M(x, y) \quad \text { and } \quad A_{b}\left(y_{0}, y_{0}, \ldots, y_{0}, T x_{1}\right) \leq r(1-h)
$$

where

$$
\begin{aligned}
M(x, y)= & \max \left\{A_{b}(f x, f x, \ldots, f x, g y), A_{b}(f x, f x, \ldots, f x, S x), A_{b}(g y, g y, \ldots, g y, T y),\right. \\
& \left.A_{b}(S x, S x, \ldots, S x, g y), A_{b}(f x, f x, \ldots, f x, T y)\right\} .
\end{aligned}
$$

Then $(f, S)$ and $(g, T)$ have a unique point of coincidence in $Y$. Furthermore, if $(f, S)$ and $(g, T)$ are weakly compatible, then there exists a unique common fixed point of $f, T, S$, and $g$ in $Y$.

Proof Since $T(Y) \subseteq f(Y)$, there exists a point $x_{2}$ in $Y$ such that $y_{1}=T\left(x_{1}\right)=f\left(x_{2}\right), \ldots$, so there exist $\left\{x_{k}\right\}$ and $\left\{y_{k}\right\}$ in $Y$ such that $y_{2 k}=S x_{2 k}=g x_{2 k+1}$ and $y_{2 k+1}=T x_{2 k+1}=f x_{2 k+2}$ for all $k$ in $\mathbb{N}_{0}$.

Now we show that $y_{k} \in Y$ for all $k \in \mathbb{N}$.

Since $A_{b}\left(y_{0}, y_{0}, \ldots, y_{0}, T x_{1}\right)=A_{b}\left(y_{0}, y_{0}, \ldots, y_{0}, y_{1}\right) \leq r(1-h)<r$, it follows that $y_{1} \in Y$. Suppose that $\left\{y_{2}, y_{3}, \ldots, y_{j}\right\} \subseteq Y$ for some $j \in \mathbb{N}$. If $j=2 t$, then by (2.1) we have

$$
\begin{aligned}
& A_{b}\left(y_{2 t}, y_{2 t}, \ldots, y_{2 t}, y_{2 t+1}\right) \\
&=A_{b}\left(S x_{2 t}, S x_{2 t}, \ldots, S x_{2 t}, T x_{2 t+1}\right) \\
& \leq \alpha\left(\operatorname { m a x } \left\{A_{b}\left(f x_{2 t}, f x_{2 t}, \ldots, f x_{2 t}, g x_{2 t+1}\right), A_{b}\left(f x_{2 t}, f x_{2 t}, \ldots, f x_{2 t}, S x_{2 t}\right),\right.\right. \\
& A_{b}\left(g x_{2 t+1}, g x_{2 t+1}, \ldots, g x_{2 t+1}, T x_{2 t+1}\right), A_{b}\left(S x_{2 t}, S x_{2 t}, \ldots, S x_{2 t}, g x_{2 t+1}\right), \\
&\left.\left.A_{b}\left(f x_{2 t}, f x_{2 t}, \ldots, f x_{2 t}, T x_{2 t+1}\right)\right\}\right) \\
&= \alpha\left(\operatorname { m a x } \left\{A_{b}\left(y_{2 t-1}, y_{2 t-1}, \ldots, y_{2 t-1}, y_{2 t}\right), A_{b}\left(y_{2 t-1}, y_{2 t-1}, \ldots, y_{2 t-1}, y_{2 t}\right),\right.\right. \\
& A_{b}\left(y_{2 t}, y_{2 t}, \ldots, y_{2 t}, y_{2 t+1}\right), A_{b}\left(y_{2 t}, y_{2 t}, \ldots, y_{2 t}, y_{2 t}\right), \\
&\left.\left.A_{b}\left(y_{2 t-1}, y_{2 t-1}, \ldots, y_{2 t-1}, y_{2 t+1}\right)\right\}\right) \\
&= \alpha\left(\operatorname { m a x } \left\{A_{b}\left(y_{2 t-1}, y_{2 t-1}, \ldots, y_{2 t-1}, y_{2 t}\right), A_{b}\left(y_{2 t}, y_{2 t}, \ldots, y_{2 t}, y_{2 t+1}\right),\right.\right. \\
&\left.\left.A_{b}\left(y_{2 t-1}, y_{2 t-1}, \ldots, y_{2 t-1}, y_{2 t+1}\right)\right\}\right) \\
& \leq \alpha\left(\operatorname { m a x } \left\{A_{b}\left(y_{2 t-1}, y_{2 t-1}, \ldots, y_{2 t-1}, y_{2 t}\right), A_{b}\left(y_{2 t}, y_{2 t}, \ldots, y_{2 t}, y_{2 t+1}\right),\right.\right. \\
&\left.\left.s\left[(n-1) A_{b}\left(y_{2 t-1}, y_{2 t-1}, \ldots, y_{2 t-1}, y_{2 t}\right)+A_{b}\left(y_{2 t}, y_{2 t}, \ldots, y_{2 t}, y_{2 t+1}\right)\right]\right\}\right) \\
&= \alpha s\left[(n-1) A_{b}\left(y_{2 t-1}, y_{2 t-1}, \ldots, y_{2 t-1}, y_{2 t}\right)+A_{b}\left(y_{2 t}, y_{2 t}, \ldots, y_{2 t}, y_{2 t+1}\right)\right] \\
& \leq \alpha s(n-1)\left[A_{b}\left(y_{2 t-1}, y_{2 t-1}, \ldots, y_{2 t-1}, y_{2 t}\right)+A_{b}\left(y_{2 t}, y_{2 t}, \ldots, y_{2 t}, y_{2 t+1}\right)\right] .
\end{aligned}
$$

Thus, we obtain that $A_{b}\left(y_{2 t}, y_{2 t}, \ldots, y_{2 t}, y_{2 t+1}\right) \leq h\left(A_{b}\left(y_{2 t-1}, y_{2 t-1}, \ldots, y_{2 t-1}, y_{2 t}\right)\right)$, where $h=$ $\frac{\gamma}{1-\gamma}$. Similarly, for $j=2 t+1$, we have $A_{b}\left(y_{2 t+1}, y_{2 t+1}, \ldots, y_{2 t+1}, y_{2 t+2}\right) \leq h A_{b}\left(y_{2 t}, y_{2 t}, \ldots\right.$, $\left.y_{2 t}, y_{2 t+1}\right)$. Thus,

$$
A_{b}\left(y_{t}, y_{t}, \ldots, y_{t}, y_{t+1}\right) \leq h A_{b}\left(y_{t-1}, y_{t-1}, \ldots, y_{t-1}, y_{t}\right) \quad \text { for each } t \in \mathbb{N} \text {. }
$$


Note that

$$
\begin{aligned}
A_{b}\left(y_{t}, y_{t}, \ldots, y_{t}, y_{t+1}\right) & \leq h A_{b}\left(y_{t-1}, y_{t-1}, \ldots, y_{t-1}, y_{t}\right) \\
& \leq h^{2} A_{b}\left(y_{t-2}, y_{t-2}, \ldots, y_{t-2}, y_{t-1}\right) \\
& \vdots \\
& \leq h^{t} A_{b}\left(y_{0}, y_{0}, \ldots, y_{0}, y_{1}\right) \quad \text { for each } t \in \mathbb{N} .
\end{aligned}
$$

By Lemma 1.7 we have

$$
\begin{aligned}
A_{b}\left(y_{0}, y_{0}, \ldots, y_{0}, y_{t+1}\right) \leq & s\left[(n-1) A_{b}\left(y_{0}, y_{0}, \ldots, y_{0}, y_{1}\right)\right. \\
& +h(n-1) A_{b}\left(y_{0}, y_{0}, \ldots, y_{0}, y_{1}\right) \\
& +h^{2}(n-1) A_{b}\left(y_{0}, y_{0}, \ldots, y_{0}, y_{1}\right) \\
& \vdots \\
& \left.+(n-1) h^{t-1} A_{b}\left(y_{0}, y_{0}, \ldots, y_{0}, y_{1}\right)+h^{t} A_{b}\left(y_{0}, y_{0}, \ldots, y_{0}, y_{1}\right)\right] \\
\leq & s(n-1)\left(h^{0}+h^{1}+h^{2}+\cdots+h^{t}\right) A_{b}\left(y_{0}, y_{0}, \ldots, y_{0}, y_{1}\right) \\
= & s(n-1) \frac{1-h^{t+1}}{1-h} A_{b}\left(y_{0}, y_{0}, \ldots, y_{0}, y_{1}\right) \\
\leq & r \quad \text { for each } t \in \mathbb{N} .
\end{aligned}
$$

That is, $y_{t+1} \in Y$. By induction, $\left\{y_{k}\right\} \subseteq Y$. Now we show that $\left\{y_{k}\right\}$ is a Cauchy sequence in $Y$.

For this, let $m, k \in \mathbb{N}$ with $m>k$. By Lemma 1.7 we have

$$
\begin{aligned}
A_{b}\left(y_{k}, y_{k}, \ldots, y_{k}, y_{m}\right) \leq & s\left[(n-1) A_{b}\left(y_{k}, y_{k}, \ldots, y_{k}, y_{k+1}\right)\right. \\
& +(n-1) A_{b}\left(y_{k+1}, y_{k+1}, \ldots, y_{k+1}, y_{k+2}\right) \\
& \vdots \\
& +(n-1) A_{b}\left(y_{m-2}, y_{m-2}, \ldots, y_{m-2}, y_{m-1}\right) \\
& \left.+A_{b}\left(y_{m-1}, y_{m-1}, \ldots, y_{m-1}, y_{m}\right)\right] \\
\leq & s\left[(n-1) h^{k} A_{b}\left(y_{0}, y_{0}, \ldots, y_{0}, y_{1}\right)\right. \\
& +(n-1) h^{k+1} A_{b}\left(y_{0}, y_{0}, \ldots, y_{0}, y_{1}\right) \\
& \vdots \\
& \left.+(n-1) h^{m-2} A_{b}\left(y_{0}, y_{0}, \ldots, y_{0}, y_{1}\right)+h^{m-1} A_{b}\left(y_{0}, y_{0}, \ldots, y_{0}, y_{1}\right)\right] \\
\leq & (n-1) s\left(h^{k}+h^{k+1}+\ldots+h^{m-1}\right) A_{b}\left(y_{0}, y_{0}, \ldots, y_{0}, y_{1}\right) \\
\leq & (n-1) s\left(h^{k}+h^{k+1}+\ldots\right) A_{b}\left(y_{0}, y_{0}, \ldots, y_{0}, y_{1}\right) \\
= & (n-1) s\left(\frac{h^{k}}{1-h}\right) A_{b}\left(y_{0}, y_{0}, \ldots, y_{0}, y_{1}\right) .
\end{aligned}
$$

Hence, $\lim _{k, m \rightarrow \infty} A_{b}\left(y_{k}, y_{k}, y_{k}, \ldots, y_{k}, y_{m}\right)=0$, that is, $\left\{y_{k}\right\}$ is a Cauchy sequence in $Y$. 
Next, we assume that $T(Y)$ is a complete subspace of $Y$. We choose a point $x^{*}$ in $T(Y)$ such that $\lim _{k \rightarrow \infty} y_{k}=x^{*}$. Then it follows that

$$
\lim _{k \rightarrow \infty} S x_{2 k}=\lim _{k \rightarrow \infty} g x_{2 k+1}=\lim _{k \rightarrow \infty} T x_{2 k+1}=\lim _{k \rightarrow \infty} f x_{2 k+2}=x^{*}
$$

Since $T(Y) \subseteq f(Y)$, there exists a point $y$ in $Y$ such that $f(y)=x^{*}$. It follows from (2.1) that

$$
\begin{aligned}
A_{b}\left(S y, S y, \ldots, S y, x^{*}\right) \leq & (n-1) s A_{b}\left(S y, S y, \ldots, S y, T x_{2 k+1}\right) \\
& +s A_{b}\left(T x_{2 k+1}, T x_{2 k+1}, \ldots, T x_{2 k+1}, x^{*}\right) \\
\leq & \gamma\left(M\left(y, x_{2 k+1}\right)\right)+s A_{b}\left(T x_{2 k+1}, T x_{2 k+1}, \ldots, T x_{2 k+1}, x^{*}\right),
\end{aligned}
$$

where

$$
\begin{aligned}
M\left(y, x_{2 k+1}\right)= & \max \left\{A_{b}\left(f y, f y, \ldots, f y, g x_{2 k+1}\right), A_{b}(f y, f y, \ldots, f y, S y),\right. \\
& A_{b}\left(g x_{2 k+1}, g x_{2 k+1}, \ldots, g x_{2 k+1}, T x_{2 k+1}\right), A_{b}\left(S y, S y, \ldots, S y, g x_{2 k+1}\right), \\
& \left.A_{b}\left(f y, f y, \ldots, f y, T x_{2 k+1}\right)\right\} \\
= & \max \left\{A_{b}\left(x^{*}, x^{*}, \ldots, x^{*}, g x_{2 k+1}\right), A_{b}\left(x^{*}, x^{*}, \ldots, x^{*}, S y\right),\right. \\
& A_{b}\left(g x_{2 k+1}, g x_{2 k+1}, \ldots, g x_{2 k+1}, T x_{2 k+1}\right), A_{b}\left(S y, S y, \ldots, S y, g x_{2 k+1}\right), \\
& \left.A_{b}\left(x^{*}, x^{*}, \ldots, x^{*}, T x_{2 k+1}\right)\right\} .
\end{aligned}
$$

We consider the following cases:

(i) If $M\left(y, x_{2 k+1}\right)=A_{b}\left(x^{*}, x^{*}, \ldots, x^{*}, g x_{2 k+1}\right)$, then we have

$$
\begin{aligned}
& A_{b}\left(S y, S y, \ldots, S y, x^{*}\right) \\
& \quad \leq \gamma A_{b}\left(x^{*}, x^{*}, \ldots, x^{*}, g x_{2 k+1}\right)+s A_{b}\left(T x_{2 k+1}, T x_{2 k+1}, \ldots, T x_{2 k+1}, x^{*}\right) .
\end{aligned}
$$

Taking the limit as $k \rightarrow \infty$, we have

$$
A_{b}\left(S y, S y, \ldots, S y, x^{*}\right) \leq \gamma A_{b}\left(x^{*}, x^{*}, \ldots, x^{*}, x^{*}\right)=0 .
$$

Hence, $A_{b}\left(S y, S y, \ldots, S y, x^{*}\right)=A_{b}\left(x^{*}, x^{*}, \ldots, x^{*}, S y\right)=0$ implies that $S y=x^{*}$.

(ii) If $M\left(y, x_{2 k+1}\right)=A_{b}\left(x^{*}, x^{*}, \ldots, x^{*}, S y\right)$, then we obtain that

$$
\begin{aligned}
& A_{b}\left(S y, S y, \ldots, S y, x^{*}\right) \\
& \quad \leq \gamma A_{b}\left(S y, S y, \ldots, S y, x^{*}\right)+s A_{b}\left(T x_{2 k+1}, T x_{2 k+1}, \ldots, T x_{2 k+1}, x^{*}\right) .
\end{aligned}
$$

Taking the limit as $k \rightarrow \infty$, we have

$$
A_{b}\left(S y, S y, \ldots, S y, x^{*}\right) \leq \gamma A_{b}\left(S y, S y, \ldots, S y, x^{*}\right),
$$

which further implies that $S y=x^{*}$. 
(iii) When $M\left(y, x_{2 k+1}\right)=A_{b}\left(g x_{2 k+1}, g x_{2 k+1}, \ldots, g x_{2 k+1}, T x_{2 k+1}\right)$, we have

$$
\begin{aligned}
A_{b}\left(S y, S y, \ldots, S y, x^{*}\right) \leq & \gamma A_{b}\left(g x_{2 k+1}, g x_{2 k+1}, \ldots, g x_{2 k+1}, T x_{2 k+1}\right) \\
& +s A_{b}\left(T x_{2 k+1}, T x_{2 k+1}, \ldots, T x_{2 k+1}, x^{*}\right),
\end{aligned}
$$

which by taking the limit as $k \rightarrow \infty$ gives that

$$
A_{b}\left(S y, S y, \ldots, S y, x^{*}\right) \leq \gamma d\left(x^{*}, x^{*}, \ldots, x^{*}, x^{*}\right)=0
$$

and hence $S y=x^{*}$.

(iv) If $M\left(y, x_{2 k+1}\right)=A_{b}\left(S y, S y, \ldots, S y, g x_{2 k+1}\right)$, then we have

$$
\begin{aligned}
& A_{b}\left(S y, S y, \ldots, S y, x^{*}\right) \\
& \quad \leq \gamma A_{b}\left(S y, S y, \ldots, S y, g x_{2 k+1}\right)+s A_{b}\left(T x_{2 k+1}, T x_{2 k+1}, \ldots, T x_{2 k+1}, x^{*}\right) .
\end{aligned}
$$

Taking the limit as $k \rightarrow \infty$, we obtain that

$$
A_{b}\left(S y, S y, \ldots, S y, x^{*}\right) \leq \gamma A_{b}\left(S y, S y, \ldots, S y, x^{*}\right),
$$

which implies that $S y=x^{*}$.

(v) When $M\left(y, x_{2 k+1}\right)=A_{b}\left(x^{*}, x^{*}, \ldots, x^{*}, T x_{2 k+1}\right)$, we have

$$
\begin{aligned}
& A_{b}\left(S y, S y, \ldots, S y, x^{*}\right) \\
& \quad \leq \gamma A_{b}\left(x^{*}, x^{*}, \ldots, x^{*}, T x_{2 k+1}\right)+s A_{b}\left(T x_{2 k+1}, T x_{2 k+1}, \ldots, T x_{2 k+1}, x^{*}\right),
\end{aligned}
$$

which by taking the limit as $k \rightarrow \infty$ implies that

$$
A_{b}\left(S y, S y, \ldots, S y, x^{*}\right) \leq \gamma A_{b}\left(x^{*}, x^{*}, \ldots, x^{*}, x^{*}\right),
$$

and hence $S y=x^{*}$.

Thus, in all cases, we have $S y=x^{*}$, and hence $S y=f y=x^{*}$ is a unique coincidence point of $(f, S)$ in $Y$.

Since $S(Y) \subseteq g(Y)$, we choose a point $z$ in $Y$ such that $g(z)=x^{*}$. Note that

$$
\begin{aligned}
A_{b}\left(T z, T z, \ldots, T z, x^{*}\right) \leq & (n-1) s A_{b}\left(S x_{2 k}, S x_{2 k}, \ldots, S x_{2 k}, T z\right) \\
& +s A_{b}\left(S x_{2 k}, S x_{2 k}, \ldots, S x_{2 k}, x^{*}\right) \\
\leq & \gamma\left(M\left(x_{2 k}, z\right)\right)+s A_{b}\left(S x_{2 k}, S x_{2 k}, \ldots, S x_{2 k}, x^{*}\right),
\end{aligned}
$$

where

$$
\begin{aligned}
M\left(x_{2 k}, z\right)= & \max \left\{A_{b}\left(f x_{2 k}, f x_{2 k}, \ldots, f x_{2 k}, g z\right), A_{b}\left(f x_{2 k}, f x_{2 k}, \ldots, f x_{2 k}, S x_{2 k}\right),\right. \\
& A_{b}(g z, g z, \ldots, g z, T z), A_{b}\left(S x_{2 k}, S x_{2 k}, \ldots, S x_{2 k}, g z\right) \\
& \left.A_{b}\left(f x_{2 k}, f x_{2 k}, \ldots, f x_{2 k}, T z\right)\right\}
\end{aligned}
$$




$$
\begin{aligned}
= & \max \left\{A_{b}\left(x^{*}, x^{*}, \ldots, x^{*}, f x_{2 k}\right), A_{b}\left(f x_{2 k}, f x_{2 k}, \ldots, f x_{2 k}, S x_{2 k}\right),\right. \\
& A_{b}\left(x^{*}, x^{*}, \ldots, x^{*}, T z\right), A_{b}\left(S x_{2 k}, S x_{2 k}, \ldots, S x_{2 k}, x^{*}\right), \\
& \left.A_{b}\left(f x_{2 k}, f x_{2 k}, \ldots, f x_{2 k}, T z\right)\right\} .
\end{aligned}
$$

We again consider the following cases:

(i) If $M\left(x_{2 k}, z\right)=A_{b}\left(x^{*}, x^{*}, \ldots, x^{*}, f x_{2 k}\right)$, then we have

$$
A_{b}\left(x^{*}, x^{*}, \ldots, x^{*}, T z\right) \leq \gamma A_{b}\left(x^{*}, x^{*}, \ldots, x^{*}, f x_{2 k}\right)+s A_{b}\left(S x_{2 k}, S x_{2 k}, \ldots, S x_{2 k}, x^{*}\right),
$$

which by taking the limit as $k \rightarrow \infty$ implies $A_{b}\left(x^{*}, x^{*}, \ldots, x^{*}, T z\right) \leq \gamma A_{b}\left(x^{*}, x^{*}, \ldots, x^{*}, x^{*}\right)+$ $s A_{b}\left(x^{*}, x^{*}, \ldots, x^{*}, x^{*}\right)$, and hence $T z=x^{*}$.

(ii) If $M\left(x_{2 k}, z\right)=A_{b}\left(f x_{2 k}, f x_{2 k}, \ldots, f x_{2 k}, S x_{2 k}\right)$, then we have

$$
\begin{aligned}
& A_{b}\left(x^{*}, x^{*}, \ldots, x^{*}, T z\right) \\
& \quad \leq \gamma A_{b}\left(f x_{2 k}, f x_{2 k}, \ldots, f x_{2 k}, S x_{2 k}\right)+s A_{b}\left(S x_{2 k}, S x_{2 k}, \ldots, S x_{2 k}, x^{*}\right),
\end{aligned}
$$

which by taking the limit as $k \rightarrow \infty$ implies that

$$
\begin{aligned}
& A_{b}\left(x^{*}, x^{*}, \ldots, x^{*}, T z\right) \\
& \quad \leq \gamma A_{b}\left(x^{*}, x^{*}, \ldots, x^{*}, x^{*}\right)+s A_{b}\left(x^{*}, x^{*}, \ldots, x^{*}, x^{*}\right),
\end{aligned}
$$

which further implies that $T z=x^{*}$.

(iii) If $M\left(x_{2 k}, z\right)=A_{b}\left(x^{*}, x^{*}, \ldots, x^{*}, T z\right)$, then we have

$$
\begin{aligned}
& A_{b}\left(x^{*}, x^{*}, \ldots, x^{*}, T z\right) \\
& \quad \leq \gamma A_{b}\left(x^{*}, x^{*}, \ldots, x^{*}, T z\right)+s A_{b}\left(S x_{2 k}, S x_{2 k}, \ldots, S x_{2 k}, x^{*}\right),
\end{aligned}
$$

which by taking the limit as $k \rightarrow \infty$ gives

$$
A_{b}\left(x^{*}, x^{*}, \ldots, x^{*}, T z\right) \leq \gamma A_{b}\left(x^{*}, x^{*}, \ldots, x^{*}, T z\right)+s A_{b}\left(x^{*}, x^{*}, \ldots, x^{*}, x^{*}\right),
$$

and hence $T z=x^{*}$.

(iv) When $M\left(x_{2 k}, z\right)=A_{b}\left(S x_{2 k}, S x_{2 k}, \ldots, S x_{2 k}, x^{*}\right)$, we have

$$
\begin{aligned}
& A_{b}\left(x^{*}, x^{*}, \ldots, x^{*}, T z\right) \\
& \quad \leq \gamma A_{b}\left(S x_{2 k}, S x_{2 k}, \ldots, S x_{2 k}, x^{*}\right)+s A_{b}\left(S x_{2 k}, S x_{2 k}, \ldots, S x_{2 k}, x^{*}\right) .
\end{aligned}
$$

Taking the limit as $k \rightarrow \infty$, we obtain that

$$
\begin{aligned}
& A_{b}\left(x^{*}, x^{*}, \ldots, x^{*}, T z\right) \\
& \quad \leq \gamma A_{b}\left(x^{*}, x^{*}, \ldots, x^{*}, x^{*}\right)+s A_{b}\left(x^{*}, x^{*}, \ldots, x^{*}, x^{*}\right),
\end{aligned}
$$

and so $T z=x^{*}$. 
(v) If $M\left(x_{2 k}, z\right)=A_{b}\left(f x_{2 k}, f x_{2 k}, \ldots, f x_{2 k}, T z\right)$, then we have

$$
\begin{aligned}
& A_{b}\left(x^{*}, x^{*}, \ldots, x^{*}, T z\right) \\
& \quad \leq \gamma A_{b}\left(f x_{2 k}, f x_{2 k}, \ldots, f x_{2 k}, T z\right)+s A_{b}\left(S x_{2 k}, S x_{2 k}, \ldots, S x_{2 k}, x^{*}\right),
\end{aligned}
$$

which by taking the limit as $k \rightarrow \infty$ gives

$$
\begin{aligned}
& A_{b}\left(x^{*}, x^{*}, \ldots, x^{*}, T z\right) \\
& \quad \leq \gamma A_{b}\left(x^{*}, x^{*}, \ldots, x^{*}, T z\right)+s A_{b}\left(x^{*}, x^{*}, \ldots, x^{*}, x^{*}\right),
\end{aligned}
$$

and hence $T z=x^{*}$.

Thus, in all cases, we have $T z=g z=x^{*}$, a unique coincidence point of $(g, T)$ in $Y$.

Suppose that $(f, S)$ is weakly compatible. Then $S f y=f S y$ implies that $S x^{*}=f x^{*}$. Note that

$$
A_{b}\left(S x^{*}, S x^{*}, \ldots, S x^{*}, x^{*}\right)=A_{b}\left(S x^{*}, S x^{*}, \ldots, S x^{*}, T z\right) \leq \alpha\left(M\left(x^{*}, z\right)\right),
$$

where

$$
\begin{aligned}
M\left(x^{*}, z\right)= & \max \left\{A_{b}\left(f x^{*}, f x^{*}, \ldots, f x^{*}, g z\right), A_{b}\left(f x^{*}, f x^{*}, \ldots, f x^{*}, S x^{*}\right),\right. \\
& A_{b}(g z, g z, \ldots, g z, T z), A_{b}\left(S x^{*}, S x^{*}, \ldots, S x^{*}, g z\right), \\
& \left.A_{b}\left(f x^{*}, f x^{*}, \ldots, f x^{*}, T z\right)\right\} \\
= & \max \left\{A_{b}\left(S x^{*}, S x^{*}, \ldots, S x^{*}, x^{*}\right), A_{b}\left(f x^{*}, f x^{*}, \ldots, f x^{*}, S x^{*}\right),\right. \\
& A_{b}\left(x^{*}, x^{*}, \ldots, x^{*}, x^{*}\right), A_{b}\left(S x^{*}, S x^{*}, \ldots, S x^{*}, x^{*}\right), \\
& \left.A_{b}\left(S x^{*}, S x^{*}, \ldots, S x^{*}, x^{*}\right)\right\} .
\end{aligned}
$$

Thus, $A_{b}\left(S x^{*}, S x^{*}, \ldots, S x^{*}, x^{*}\right) \leq \alpha A_{b}\left(S x^{*}, S x^{*}, \ldots, S x^{*}, x^{*}\right)$ implies that $x^{*}=S x^{*}=f x^{*}$.

Similarly, if $(g, T)$ is weakly compatible, then $T g z=g T z$ implies that $T x^{*}=g x^{*}$. By (2.1) we have

$$
A_{b}\left(x^{*}, x^{*}, \ldots, x^{*}, T x^{*}\right)=A_{b}\left(S x^{*}, S x^{*}, \ldots, S x^{*}, T x^{*}\right) \leq \alpha\left(M\left(x^{*}, x^{*}\right)\right),
$$

where

$$
\begin{aligned}
M\left(x^{*}, x^{*}\right)= & \max \left\{A_{b}\left(f x^{*}, f x^{*}, \ldots, f x^{*}, g x^{*}\right), A_{b}\left(f x^{*}, f x^{*}, \ldots, f x^{*}, S x^{*}\right),\right. \\
& A_{b}\left(g x^{*}, g x^{*}, \ldots, g x^{*}, T x^{*}\right), A_{b}\left(S x^{*}, S x^{*}, \ldots, S x^{*}, g x^{*}\right), \\
& \left.A_{b}\left(f x^{*}, f x^{*}, \ldots, f x^{*}, T x^{*}\right)\right\} \\
= & \max \left\{A_{b}\left(x^{*}, x^{*}, \ldots, x^{*}, T x^{*}\right), A_{b}\left(f x^{*}, f x^{*}, \ldots, f x^{*}, S x^{*}\right),\right. \\
& A_{b}\left(T x^{*}, T x^{*}, \ldots, T x^{*}, T x^{*}\right), A_{b}\left(x^{*}, x^{*}, \ldots, x^{*}, T x^{*}\right), \\
& \left.A_{b}\left(x^{*}, x^{*}, \ldots, x^{*}, T x^{*}\right)\right\} .
\end{aligned}
$$

Thus, we have $A_{b}\left(x^{*}, x^{*}, \ldots, x^{*}, T x^{*}\right) \leq \alpha A_{b}\left(x^{*}, x^{*}, \ldots, x^{*}, T x^{*}\right)$, which implies that $x^{*}=$ $T x^{*}=g x^{*}$, and hence $x^{*}$ is a common fixed point of $f, T, S$, and $g$ in $Y$. 
To prove the uniqueness, let $u \in Y$ be such that $f u=g u=S u=T u=u$. Note that

$$
A_{b}\left(x^{*}, x^{*}, \ldots, x^{*}, u\right)=A_{b}\left(S x^{*}, S x^{*}, \ldots, S x^{*}, T u\right) \leq \alpha\left(M\left(x^{*}, u\right)\right)
$$

where

$$
\begin{aligned}
M\left(x^{*}, u\right)= & \max \left\{A_{b}\left(f x^{*}, f x^{*}, \ldots, f x^{*}, g u\right), A_{b}\left(f x^{*}, f x^{*}, \ldots, f x^{*}, S x^{*}\right),\right. \\
& A_{b}(g u, g u, \ldots, g u, T u), A_{b}\left(S x^{*}, S x^{*}, \ldots, S x^{*}, g u\right), \\
& \left.A_{b}\left(f x^{*}, f x^{*}, \ldots, f x^{*}, T u\right)\right\} \\
= & \max \left\{A_{b}\left(x^{*}, x^{*}, \ldots, x^{*}, u\right), A_{b}\left(x^{*}, x^{*}, \ldots, x^{*}, x^{*}\right), A_{b}(u, u, \ldots, u, u),\right. \\
& \left.A_{b}\left(x^{*}, x^{*}, \ldots, x^{*}, u\right), A_{b}\left(u, u, \ldots, u, x^{*}\right)\right\} .
\end{aligned}
$$

Hence, $x^{*}=u$.

Corollary 2.2 Let $f, g, T$, and $S$ be self-mappings of a dislocated $A_{b}$-quasi-metric space $X$ with coefficient $s \geq 1$. Assume that $S(X) \subseteq g(X), T(X) \subseteq f(X)$, and one of $S(X), g(X), T(X)$, or $f(X)$ is a complete subspace of $X$. Suppose that there exists $\alpha \in\left[0, \frac{1}{2}\right)$ such that for any $x, y \in X$, we have

$$
A_{b}(S x, S x, \ldots, S x, T y) \leq \alpha(M(x, y))
$$

where

$$
\begin{aligned}
M(x, y)= & \max \left\{A_{b}(f x, f x, \ldots, f x, g y), A_{b}(f x, f x, \ldots, f x, S x), A_{b}(g y, g y, \ldots, g y, T y),\right. \\
& \left.A_{b}(S x, S x, \ldots, S x, g y), A_{b}(f x, f x, \ldots, f x, T y)\right\} .
\end{aligned}
$$

Then $(f, S)$ and $(g, T)$ have a unique point of coincidence in $X$. Furthermore, if $(f, S)$ and $(g, T)$ are weakly compatible, then there exists a unique common fixed point of $f, T, S$, and gin $X$.

Example 2.3 Let $X$ and $A_{b}$ be as in Example 1.3. Define the mappings $f, g, S$, and $T: X \longrightarrow$ $X$ by

$$
f(x)=\left\{\begin{array}{ll}
x, & x<2, \\
2, & x \geq 2 ;
\end{array} \quad S(x)=\left\{\begin{array}{ll}
\frac{2 x}{(1+x)} & \text { if } x \neq-1, \\
0, & x=-1 ;
\end{array} \quad g(x)=T(x)= \begin{cases}\frac{x}{6} & \text { if } x \neq 1 \\
1, & x=1 .\end{cases}\right.\right.
$$

Clearly, $(f, S)$ and $(g, T)$ are pairwise weakly compatible on $X$ with $S(X) \subseteq g(X), T(X) \subseteq$ $f(X)$, and $S(X)$ a complete subspace of $X$. We now show that for all $x, y \in X$, condition (2.2) is satisfied. For this, we consider the following cases:

(i) If $x \neq 1$ and $y=1$, then

$$
\begin{aligned}
A_{b}(S x, S x, \ldots, S x, T y) & =\left|\frac{2 x}{1+x}\right|^{2}+(n-1)\left|2\left(\frac{2 x}{1+x}\right)-1\right|^{2} \\
& \leq \frac{1}{36} A_{b}(f x, f x, \ldots, f x, T y) \leq \alpha M(x, y) .
\end{aligned}
$$


(ii) If $x \neq 1$ and $y \neq 1$, then

$$
\begin{aligned}
A_{b}(S x, S x, \ldots, S x, T y) & =\left|\frac{2 x}{1+x}\right|^{2}+(n-1)\left|2\left(\frac{2 x}{1+x}\right)-\frac{y}{6}\right|^{2} \\
& \leq \frac{1}{36} A_{b}(f x, f x, \ldots, f x, T y) \leq \alpha M(x, y) .
\end{aligned}
$$

(iii) If $x=-1$ and $y=1$, then

$$
\begin{aligned}
A_{b}(S x, S x, \ldots, S x, T y) & =(n-1)|0-1|^{2} \\
& \leq \frac{1}{36} A_{b}(f x, f x, \ldots, f x, T y) \leq \alpha M(x, y) .
\end{aligned}
$$

(iv) If $x=-1$ and $y \neq 1$, then

$$
\begin{aligned}
A_{b}(S x, S x, \ldots, S x, T y) & =(n-1)\left|0-\frac{y}{6}\right|^{2} \\
& \leq \frac{1}{36} A_{b}(f x, f x, \ldots, f x, T y) \leq \alpha M(x, y) .
\end{aligned}
$$

Thus, all the conditions of Corollary 2.2 are satisfied. Moreover, $x=1$ is a common fixed point of $f, T, S$, and $g$.

\section{Competing interests}

The authors declare that they have no competing interests.

\section{Authors' contributions}

All authors contributed equally and significantly in writing this paper. All authors read and approved the final manuscript.

\section{Author details}

'Department of Mathematics and Applied Mathematics, University of Pretoria, Hatfield, Pretoria, South Africa.

${ }^{2}$ Department of Mathematics, Bayero University Kano, P.M.B. 3011, Kano, Nigeria. ${ }^{3}$ Department of Mathematics, Kano University of Science and Technology, P.M.B. 3042, Wudil, Kano, Nigeria.

Received: 19 October 2015 Accepted: 24 December 2015 Published online: 07 January 2016

\section{References}

1. Scott, DS: Domains for Denotational Semantics. Lecture Notes in Computer Science, vol. 140 (1982)

2. Matthews, SG: Partial metric topology. In: Proceedings of the 8th Summer Conference on General Topology and Applications, Flushing, NY, 1992. Annals of the New York Academy of Sciences, vol. 728, pp. 183-197. N.Y. Acad. Sci., New York (1994)

3. Arshad, M, Shoaib, A, Beg, I: Fixed point of a pair of contractive dominated mappings on a closed ball in an ordered dislocated metric space. Fixed Point Theory Appl. 2013, Article ID 115 (2013)

4. Abbas, M, Ali, B, Suleiman, Yl: Generalized common fixed point results in partially ordered A-metric spaces. Fixed Point Theory Appl. 2015, Article ID 84 (2015)

5. Hussain, N, Roshan, JR, Parvaneh, V, Latif, A: A unification of G-metric, partial metric, and b-metric spaces. Abstr. Appl. Anal. 2014, Article ID 180698 (2014). doi:10.1155/2014/180698 